\title{
PARASITISMO NATURAL EM MOSCAS-DAS-FRUTAS (DIPTERA: TEPHRITIDAE) NO SEMIÁRIDO DO SUDOESTE DA BAHIA, BRASIL ${ }^{1}$
}

\author{
RICARDO FALCÃO DE SÁ², MARIA APARECIDA CASTELLANI ${ }^{3}$, \\ ANTONIO SOUZA DO NASCIMENTO ${ }^{4}$, ANA ELIZABETE LOPES RIBEIRO ${ }^{5}$, \\ ALDENISE ALVES MOREIRA ${ }^{3}$
}

RESUMO - Parasitoides são importantes agentes de controle natural de tefritídeos, e os conhecimentos sobre as relações tritróficas podem subsidiar o manejo destas pragas. Este trabalho objetivou estimar índices de parasitismo em moscas-das-frutas, em 21 espécies vegetais, e identificar as espécies de parasitoides associados, nas condições do semiárido do sudoeste da Bahia. Oito hospedeiros apresentaram infestação por Anastrepha spp. e, destes, em quatro, ocorreu parasitismo superior a 20,0\%, sendo: 20,8\% (Ziziphus joazeiro L.); 21,3\% (Spondias tuberosa L.); 32,4\% (Spondias purpurea L.) e 57,1\% (Malpighia emarginata L.). Os parasitoides coletados pertencem à família Braconidae, sendo $89 \%$ de Doryctobracon areolatus e $11 \%$ de Asobara anastrephae.

Termos para indexação: Controle biológico, parasitoides, moscas-das-frutas, Doryctobracon, Asobara.

\section{NATURAL PARASITISM IN FRUIT-FLIES IN THE FRUTICULTURE AREA OF ANAGÉ, SEMI-ARID OF SOUTHWESTERN BAHIA, BRAZIL}

\begin{abstract}
Parasitoids are important natural control agents of tephritids and knowledge about the tritrophic relationships can support the management of these pests. This study aimed to estimate of parasitism indexes in fruit flies in 21 plant species and identify the species of parasitoids associated, in semiarid conditions of Southwestern Bahia. Eight hosts showed infestation by Anastrepha spp. and, of these, four occurred parasitism above $20.0 \%$, of which: 20.8\% (Ziziphus joazeiro L.); 21.3\% (Spondias tuberosa L.); 32.4\% (Spondias purpurea L.) and $57.1 \%$ (Malpighia emarginata L.). The collected parasitoids belong to the Braconidae family, $89 \%$ of Doryctobracon areolatus and $11 \%$ of Asobara anastrephae.
\end{abstract}

Index terms: Biological control, parasitoids, fruit-flies, Doryctobracon, Asobara.

O Brasil é um importante produtor de frutas no mundo, e o Estado da Bahia figura como primeiro produtor nacional de manga, com produção de 509.676 toneladas em 2010, ocupando 28.058 ha. Além dos polos tradicionais de fruticultura do Estado da Bahia, como o do Submédio São Francisco e de Livramento de Nossa Senhora, a região do Rio Gavião, localizada no semiárido do sudoeste do Estado, vem despontando como um novo polo produtor de frutíferas, especialmente manga, apresentando grande potencial para impulsionar a economia regional e estadual.
As moscas-das-frutas (Diptera: Tephritidae) constituem os principais problemas fitossanitários da cultura da manga, principalmente quando o objetivo é o mercado externo. A utilização de métodos alternativos ao controle químico tem ganhado importância nos últimos anos, na medida em que programas de Produção Integrada de Frutas (PIF) têm sido norteados pelos princípios da preservação dos recursos naturais e segurança alimentar alcançados, principalmente pela redução do uso de agrotóxicos e ausência de resíduos químicos nos alimentos. Nesse sentido, métodos de supressão populacional, como a

\footnotetext{
${ }^{1}$ (Trabalho 088-12). Recebido em: 01-02-2012. Aceito para publicação em 18-10-2012.

${ }^{2} \mathrm{Eng}^{\mathrm{o}} \mathrm{Agr}^{\mathrm{O}} \mathrm{MSc}$., Fiscal Agropecuário, Agência Estadual de Defesa Agropecuária da Bahia, ADAB, Rua Teodoro Sampaio, 423, São Vicente, Vitória da Conquista-BA. E-mail: ricardofs@adab.ba.gov.br

${ }^{3} \mathrm{Eng}^{\mathrm{o}} \mathrm{Agr}^{\circ}$ DSc., Professor, Universidade Estadual do Sudoeste da Bahia, UESB, Departamento de Fitotecnia e Zootecnia. E-mails: castellani.uesb@gmail.com, aldenise.moreira@gmail.com

${ }^{4}$ Eng $^{\circ}$. Agr ${ }^{\circ}$. DSc., Pesquisador, Empresa Brasileira de Pesquisa Agropecuária/CNPMF. E-mail: antnasc@cnpmf.embrapa.br

${ }^{5} \mathrm{Eng}^{\mathrm{o}} \mathrm{Agr}^{\circ}$ DSc., Pós-Doutoranda PNPD/CAPES, Universidade Estadual do Sudoeste da Bahia, UESB, Departamento de Fitotecnia e Zootecnia. E-mail: ana.lorib@gmail.com
} 
Técnica do Inseto Estéril (TIE) e o controle biológico, são de grande importância para o desenvolvimento sustentável da fruticultura.

Mais de 100 espécies de braconídeos (Hymenoptera: Braconidae) já foram identificados como parasitoides de tefritídeos no mundo, sendo a maioria pertencente à subfamília Opiinae (WHARTON; YODER, 2012). Parasitismo por espécies das famílias Chalcidae, Diapriidae, Eulophidae e Pteromalidae também já foi registrado (WHARTON; GILSTRAP, 1983). No Brasil, os parasitoides de moscas-das-frutas pertencem, principalmente, às famílias Braconidae, Figitidae e Pteromalidae, sendo os braconídeos mais frequentemente coletados, compreendendo treze espécies registradas. Destas, Doryctobracon areolatus (Szépligeti) é a mais frequente no Brasil (COVA; BITTENCOURT, 2003), México (LOPES et al., 1999) e Argentina (OVRUSKI et al., 2005). $\mathrm{Na}$ Bahia, região do Recôncavo, foi verificada a ocorrência do eucoilídeo Aganaspis pelleranoi (Brêthes) e dos braconídeos D. areolatus, Utetes (Braconastrepha) anastrephae (Viereck), Opius sp. e do parasitoide introduzido Diacasmimorpha longicaudata (Ashmead). Posteriormente, mais uma espécie de braconídeo de ocorrência no Estado da Bahia foi descrita, Doryctobracon fluminensis (Szépligeti). Estudos sobre relações tróficas envolvendo planta hospedeira, espécies de moscas e de seus parasitoides têm sido desenvolvidos nas regiões centro-norte (COVA;BITTENCOURT, 2003), sul (CARVALHO et al., 2004), extremo sul (BITTENCOURT et al., 2011) e sudeste (SILVA et al., 2010), existindo escassez de dados para a região sudoeste da Bahia.

O objetivo do presente trabalho foi estimar índices de parasitismo em diversos hospedeiros, nativos e exóticos, para as condições do semiárido da região sudoeste da Bahia, bem como identificar os parasitoides associados às moscas-das-frutas.

Os estudos foram desenvolvidos em pomares comerciais de manga e em suas proximidades, localizados nos municípios de Anagé (14³6'S e $\left.41^{\circ} 08^{\prime} \mathrm{W}\right)$, Belo Campo $\left(15^{\circ} 02^{\prime} \mathrm{S}\right.$ e $\left.41^{\circ} 15^{\prime} \mathrm{W}\right)$ e Caraíbas $\left(14^{\circ} 40^{\prime} \mathrm{S}\right.$ e $\left.41^{\circ} 14^{\prime} \mathrm{W}\right)$, no período de fevereiro de 2004 a dezembro de 2005 . O clima predominante na região é do tipo semiárido e subúmido a seco, com regime pluviométrico na primavera-verão.

As coletas de frutos de 21 espécies vegetais foram realizadas em função da disponibilidade dos mesmos, conforme período principal de frutificação. Procedeu-se à coleta de 0,5 a $5,0 \mathrm{~kg}$ de frutos maduros por amostra, sendo os mesmos transportados ao Laboratório de Entomologia da Universidade Estadual do Sudoeste da Bahia (UESB), com posterior contagem, pesagem e acondicionamento em bandejas plásticas, contendo vermiculita. Decorrido um período de 12 a 13 dias, os pupários foram coletados e transferidos para vidros contendo fina camada de vermiculita e fechados com tecido voile, visando à emergência de adultos. Os parasitoides foram identificados em morfoespécies, acondicionados em recipiente com álcool a 70\% e enviados ao Laboratório de Taxonomia da Escola Superior de Agricultura Luiz de Queiroz (ESALQ/ USP), Piracicaba-SP, para confirmação das espécies. $\mathrm{O}$ índice de parasitismo foi calculado pela fórmula: $\%$ de parasitismo $=$ [número de parasitoides emergidos/(número de moscas emergidas + número de parasitoides emergidos)] x 100 (PARANHOS et al., 2007).

Das 21 espécies vegetais estudadas, foram encontrados pupários de moscas-das-frutas em apenas oito espécies, com ocorrência de parasitismo em quatro (Tabela 1). Foram obtidos 1.082 pupários, com emergência de parasitoides em 71 pupários do total $(6,6 \%)$ e obtenção de índices de parasitismo superiores a $20 \%$, sendo: $20,8 \%$ em juá; $21,3 \%$ em seriguela; $32,4 \%$ em umbu e $57,1 \%$ em acerola (Tabela 1). A ausência de parasitoides nos frutos das outras espécies vegetais infestadas por moscasdas-frutas, como cajarana, goiaba, amendoeira e manga (de vez e madura), pode ter sido decorrente dos baixos índices de infestação verificados nesses hospedeiros (Tabela 1), como também pelo tamanho e morfologia dos frutos, presença de voláteis, dentre outras características, as quais, segundo Marinho et al. (2009), podem afetar o parasitismo. Frutos menores, com polpa rasa, apresentam índices de parasitismo maiores do que em frutos grandes, com polpas espessas (MARINHO et al., 2009), fato também verificado no presente trabalho.

Os parasitoides obtidos são braconídeos, pertencentes às subfamílias Opiinae e Alysiinae, representadas pelas espécies $D$. areolatus e $A$. anastrephae, respectivamente. D. areolatus ocorreu parasitando Anastrepha spp. em frutos de umbu, seriguela, juá e acerola, enquanto $A$. anastrephae ocorreu apenas em frutos de umbu e seriguela. As duas espécies já foram registradas para o Estado da Bahia. Dos espécimes obtidos, 63 (88,7\%) pertencem à espécie $D$. areolatus, confirmando tratar-se de uma espécie de ampla distribuição geográfica e mais frequente em várias regiões do Brasil (GALLI;RAMPAZZO, 2000; AGUIAR-MENEZES et al., 2001; ARAÚJO; ZUCCHI, 2002; UCHOAFERNANDES et al., 2003, MARINHO et al., 2009). 
Na Bahia, Cova e Bittencourt (2003) relataram a referida espécie como a mais frequente (54\%) na microrregião de Irecê, semiárido baiano. Em frutos de cajazeiras de Presidente Tancredo Neves-BA, do total de parasitismo encontrado por Carvalho et al. (2004), $70,78 \%$ foi por $D$. areolatus. A espécie $A$. anastrephae é também relatada por diversos autores parasitando tefritídeos no Brasil (GALLI; RAMPAZZO, 2000; UCHÔA-FERNANDES et al., 2003), contribuindo com $14,18 \%$ do total de parasitismo observado em Presidente Tancredo Neves-BA (CARVALHO et al., 2004).

Os índices de parasitismo são variáveis, de acordo com o local e os hospedeiros estudados, mas, em geral, são baixos (AGUIAR-MENEZES;MENEZES, 1997; AGUIAR-MENEZES et al., 2001). Esta afirmação também se aplica ao Estado da Bahia, onde Cova e Bittencourt (2003) observaram, na região de Irecê, índices de parasitismo de $11 \%$ em diferentes hospedeiros. Apesar disso, Aguiar-Menezes e Menezes (1997) consideram que altas taxas de parasitismo obtidas em certos hospedeiros podem ser um indicador de seleção de plantas para plantio em áreas de fruticultura, visando a promover o aumento do parasitismo natural em tefritídeos. Recentemente, Bittencourt et al. (2011) registraram a ocorrência dos parasitoides $D$. areolatus, $U$. anastraphae e Asobara anastrephae (Muesebeck) no sul da Bahia, com associações tritróficas inéditas entre frutos de sapoti, Anastrepha serpentina (Wiedemann) e A. Anastrephae e frutos de lima e amora com $A$. bahiensis e $D$. Areolatus, com índices de parasitismo de até $8,97 \%$ (amora).

Assim, no presente trabalho, registra-se a ocorrência de parasitismo natural em moscasdas-frutas no polo de fruticultura do semiárido da região sudoeste da Bahia, pelos braconídeos $D$. areolatus e $A$. anastrephae, espécies adaptadas à região e a vários hospedeiros nativos, e que devem ser consideradas em programas de manejo integrado como importantes fatores bióticos de mortalidade de Anastrepha spp..

Os autores agradecem à Fundação de Amparo à Pesquisa do Estado da Bahia - FAPESB, pelo apoio financeiro; à Agência Estadual de Defesa Agropecuária da Bahia - ADAB, pelo apoio logístico; à pesquisadora Dra. Claudia Fidelis Marinho, pela confirmação das espécies de parasitoides, e ao Programa de Pós-Graduação em Agronomia (Fitotecnia) da Universidade Estadual do Sudoeste da Bahia - UESB, pela infraestrutura disponibilizada.

TABELA 1- Hospedeiros, número de pupários de moscas-das-frutas, infestação por moscas-das-frutas, número de parasitoides e índice de parasitismo (\%) em tefritídeos. Anagé, Caraíbas e Belo Campo-BA, 2004 e 2005.

\begin{tabular}{|c|c|c|c|c|c|}
\hline Nome Científico & Nome Comum & $\begin{array}{c}\text { Pupários } \\
\left(\mathbf{N}^{0}\right)\end{array}$ & $\begin{array}{c}\text { Infestação } \\
\text { (pupário/ Kg fruto) }\end{array}$ & $\begin{array}{c}\text { Parasitoides } \\
\left(\mathbf{N}^{\circ}\right)\end{array}$ & $\begin{array}{c}\text { Indice de } \\
\text { Parasitismo } \\
(\%)\end{array}$ \\
\hline$\overline{\text { Spondias tuberosa }}$ & Umbu & 742 & 33,09 & 47 & 21,3 \\
\hline Malpighia emarginata & Acerola & 7 & 0,94 & 3 & 57,1 \\
\hline Spondias purpurea & Seriguela & 77 & 61,25 & 11 & 32,4 \\
\hline Pereskia grandfolia & Quiabento & 0 & 0 & 0 & 0 \\
\hline Spondias sp. & Cajarana & 74 & 3,46 & 0 & 0 \\
\hline Psidium guajava & Goiaba & 1 & 0,45 & 0 & 0 \\
\hline Carica papaya & Mamão & 0 & 0 & 0 & 0 \\
\hline Ziziphus joazeiro & Juá & 144 & 38,30 & 10 & 20,8 \\
\hline Annona squamosa & Pinha & 0 & 0 & 0 & 0 \\
\hline Citrus limon & Limão & 0 & 0 & 0 & 0 \\
\hline Opuntia ficus-indica & Palma & 0 & 0 & 0 & 0 \\
\hline Averrhoa carambola & Carambola & 0 & 0 & 0 & 0 \\
\hline Mormodica charantia & Melão- de -São Caetano & 0 & 0 & 0 & 0 \\
\hline Myrciaria cauliflora & Jabuticaba & 0 & 0 & 0 & 0 \\
\hline Persea americana & Abacate & 0 & 0 & 0 & 0 \\
\hline Terminalia catappa & Amendoeira & 2 & 0,14 & 0 & 0 \\
\hline Punica granatum & Romã & 0 & 0 & 0 & 0 \\
\hline Tamarindus indica & Tamarindo & 0 & 0 & 0 & 0 \\
\hline Cereus jamacaru & Mandacaru & 0 & 0 & 0 & 0 \\
\hline Ficus carica & Figo & 0 & 0 & 0 & 0 \\
\hline Mangifera indica & Manga madura & 32 & 0,07 & 0 & 0 \\
\hline Mangifera indica & Manga verde & 0 & 0 & 0 & 0 \\
\hline Mangifera indica & Manga "de vez" & 3 & 0,03 & 0 & 0 \\
\hline
\end{tabular}




\section{REFERÊNCIAS}

AGUIAR-MENEZES, E. L.; MENEZES, E. B. Natural occurrence of parasitoids of Anastrepha spp. Schiner, 1868 (Diptera: Tephritidae) in different host plants, in Itaguaí (RJ), Brazil. Biological Control, Orlando, v.8, p.1-6, 1997.

AGUIAR-MENEZES, E. L.; MENEZES, E. B.; SILVA, P. S.; BITTAR, A. C.; CASSINO, P. C. R. Native hymenopteran parasitoids associated with Anastrepha spp. (Diptera: Tephritidae) in Seropedica City, Rio de Janeiro, Brazil. Florida Entomologist, Gainesville, v.84, p.706-711, 2001.

ARAÚJO, E. L.; ZUCCHI, R. A. Hospedeiros e níveis de infestação de Neosilba pendula (Bezzi) (Diptera: Lonchaeidae) na região de Mossorá-AssuRN. Arquivos do Instituto Biológico, São Paulo, v.69, p.91-94, 2002.

BITTENCOURT, M. A. L.; SILVA, A. C. M. da; SILVA, V. E. S.; BOMFIM, Z. V.; GUIMARÃES, J.A.; SOUZA FILHO, M. F.; ARAUJO, E. L. Moscas-das-frutas (Diptera: Tephritidae) e seus parasitoides (Hymenoptera: Braconidae) associados às plantas hospedeiras no sul da Bahia. Neotropical Entomology, Londrina, v.40, n.3, p.405-406, 2011.

CARVALHO, C. A. L.; SANTOS, W. S.; DANTAS, A. C. V. L.; MARQUES, O. M. M.; PINTO, W. S. Moscas-das-frutas e parasitoides associados a frutos de cajazeiras em Presidente Tancredo Neves - Bahia. Magistra, Cruz das Almas, v.16, p.85-90, 2004.

COVA, A. K. W.; BITTEnCOURT, M. A. L. Ocorrência de moscas-das-frutas (Tephritidae) e parasitoides em frutos da região do semiárido da Bahia. Magistra, Cruz das Almas, v.15, p.67-70, 2003.

GALLI, J. S.; RAMPAZZO, E. F. Ocorrência de parasitoides emergidos de pupas de moscas-dasfrutas coletadas em goiabas no município de Monte Alto-SP. Revista de Agricultura, Piracicaba, v.75, p.109-117, 2000.
LOPES, M.; ALUJA, M. J.; SIVINSKI, M. Hymenopterous larval-pupal and pupal parasitoids of Anastrepha flies (Diptera: Tephritidae) in México. Biological Control, Orlando, v.15, p.119-129, 1999.

OVRUSKI, S. M.; WHARTON, R. A.; SCHLISERMAN, P.; ALUJA, M. Abundance of Anastrepha fraterculus (Diptera: Tephritidae) and its associated native parasitoids (Hymenoptera) in "Feral" guavas growing in the Endangered Northernmost Yungas Forests of Argentina with an Update on the taxonomic status of Opiine Parasitoids previously reported in this country. Environmental Entomology, Lanham, v.34, n.4, p.807-818, 2005.

PARANHOS, B.A.J.; WALDER, J.M.M.; ALVARENGA, C.D. Parasitismo de Larvas de Mosca-do-Mediterrâneo por Diachasmimorpha longicaudata (Ashmead) (Hymenoptera: Braconidae) em Diferentes Cultivares de Goiaba. Neotropical Entomology, Londrina, v. 36, n. 2, p.243-246, 2007.

SILVA, J.G.; DUTRA, V. S.; SANTOS, M. S.; SILVA, N. M. O.; VIDAL, D. B.; NINK, R. A.; GUIMARÃES, J. A.; ARAÚJO, E. L. Diversity of Anastrepha spp. (Diptera: Tephritidae) and associated braconid parasitoids from native and exotic host in southeastern Bahia, Brazil. Environmental Entomology, Lanham, v.39, p.1.457-1.465, 2010.

UCHÔA-FERNANDES, M. A.; MOLINA, R. M. S.; OLIVEIRA, I.; ZUCCHI, R. A.; CANAL, N. A.; DIAZ, N. B. Larval endoparasitoids (Hymenoptera) of frugivorous flies (Diptera, Tephritoidea) reared from fruits of te cerrado of the State of Mato Grosso do Sul, Brazil . Revista Brasileira de Entomologia, São Paulo, v.47, n.2, p.181-186, 2003.

WHARTON, R. A.; GILSTRAP, F. E. Key and status of Opiinae Braconid (Hymenoptera) parasitoids used in biological control of Ceratitis and Dacus s. l. (Diptera:Tephritidae). Annals of the Entomological Society of America, Lexington, v.76, p.721-746, 1983.

WHARTON, R. A.; YODER, M. J. Parasitoids of Fruit-Infesting Tephritidae. Disponível em: $<\mathrm{http}: / /$ paroffit.org >. Acesso em: 28 maio 2012. 